\title{
SESSION 2
}

Chairman: B. A. Lindblad 


\title{
7. METEOR SPECTRA
}

\author{
(Survey Paper)
}

\author{
Z. CEPLECHA \\ (Astronomical Institute of the Czechoslovak \\ Academy of Sciences, Ondrejov)
}

\begin{abstract}
1. Introduction
Meteor luminosity is frequently used for the computation of meteor mass. This procedure is a rough approximation only if the spectral distribution of the light energy and its changes during the meteor flight are not known. A good-quality photograph of a meteor spectrum includes much more information on the meteor phenomenon than any other method can provide. There is of course a stronger limitation by meteor brightness than for direct meteor photography. Detailed spectral analysis is available for meteors 4 or 5 magnitudes brighter than for those photographed directly. This could be the reason that meteor spectroscopy is not applied to a desirable extent for the study of meteor phenomenon.
\end{abstract}

\section{Observational Programs}

At present large systematic programs of meteor spectroscopy are active in Canada at the Meanook, Newbrook and Springhill Meteor Observatories, in Czechoslovakia at the Ondřejov and Skalnaté Pleso Observatories, and in the U.S.S.R. at the Ašhabad Observatory. Smaller observational programs are carried out in the U.S.S.R., U.S.A., Great Britain, Japan, The Netherlands, and India.

Slitless spectroscopy with objective prisms or gratings is the only technique used for meteors. In the past decade there has been a distinct trend from the prism spectrograph to the grating. The usual dispersions are below $100 \AA / \mathrm{mm}$, the highest ones achieved $10 \AA / \mathrm{mm}$ (Halliday, 1961, 1963; Millman and Halliday, 1961 ; Ceplecha and Rajchl, 1963; Ceplecha, 1965, 1966b). Rotating shutters are used for the separation of the wake from the head spectrum and for the determination of the dispersion direction. A special program of jumping-film cameras with low dispersion is used by Halliday and Griffin (1963) for the oxygen line 5577 in meteor spectra, and for meteor persistent-train photography. Spectra of some re-entry objects simulating 'artificial meteoroids' at low velocities $(11 \mathrm{~km} / \mathrm{sec})$ were photographed by Harvey (1967). Spectra of faint Perseids were discussed by Russell (1964). Spectra of meteor persistent trains were achieved by Nasyrova and Nasyrov (1966), starting the exposure a few 
seconds after the passage of bright meteors. Many good-quality Leonid spectra were secured by Halliday (1967), 60 spectra, and by Hirose (1967). The panchromatic region was extended by the spectra of Millman and Halliday (1961) in the infrared region to $9000 \AA$, and by Halliday (1967) in the ultraviolet region to $3150 \AA$. Special attention is being paid by Millman (1967) to a correlation of the spectroscopic results with the radar and visual data. The world list of meteor spectra has been maintained by Millman since 1934 and is currently being brought up-to-date.

\section{Identification Problems}

The main meteor luminosity (luminous head of the phenomenon) consists almost entirely of atomic emission lines belonging to neutral and ionized atoms of several elements, mainly atoms of the body itself. The atmospheric lines are only insignificant traces in the visible region, becoming more important in the infrared region. The grating spectra of dispersions of the order of $100 \AA / \mathrm{mm}$ or better make the prism spectra useless for identification problems. Any realistic identification in meteor spectra with low dispersion must be based mainly on a comparison with high-dispersion spectra. A good-quality meteor spectrum with dispersion better than $100 \AA / \mathrm{mm}$ yields more than $50 \%$ of its emission lines without blends.

The wavelengths in grating spectra can be directly computed from the coordinate system defined by the zero-order star images (Ceplecha, 1961) without previous knowledge of any identification. Thus the main emissions of meteor spectra are identified without any shadow of doubt. On the other hand the identifications of faint lines "reflect to a considerable degree, the previous experience and judgement of the particular investigator" (Halliday, 1961, p. 12). If low-dispersion prism spectrograms are used as 'independent' events for the identification, then this 'personal dependence' holds also for the brightest lines. Here is a danger for any statistics of the lines identified in meteor spectra. The low-dispersion spectrograms containing more identifications with a high 'personal factor' are in such statistics more frequent than good-quality spectra with a greater reliability of the identifications. Thus only statistics of line identification in spectra of comparable dispersion and quality are representative for the phenomenon.

There are at least four criteria one must bear in mind for identification: measured wavelength, intensity, excitation potential, and the presence of other lines of the same multiplet with corresponding intensity. It is very helpful if the intensity of a doubtful or blended line can be computed from other well-identified lines (e.g. by the method of the emission curve of growth).

\section{Line Intensities}

Measuring the wavelengths and identification is only half the work. The intensity of each line, especially of those which are without blends, is highly desirable. This is 
important even with the high-dispersion grating spectra. The change of line intensity along the meteor trajectory ought to be determined for each good-quality spectrum. The least requirement is the publication of relative total intensities of lines, i.e. the measured values corrected for selective transmission of the atmosphere, the objective and the grating, and for the spectral sensitivity of the plate. Absolute total intensities of lines are of even greater value for any quantitative study. As the calibration of the meteor plates by spectrophotometrical standards similar to the meteor image seems to be a big problem, this is usually not used in meteor spectroscopy. But there are on each plate star images, zero- and first-order ones, which are suitable for the calibration of the plate. Photometry can be carried out by a method published by Millman and Cook (1959) or by a method published by Ceplecha $(1961,1963)$ and Ceplecha and Rajchl (1963). The total intensity of a line is then expressed in ergs/sec if the distance of the particular point on the meteor trajectory is known. With the present dispersion and slitless spectrographs it is not possible to measure line profiles, which are rather the 'instrumental profiles', the monochromatic images of the radiating volume. Thus the line intensities (spectral irradiances) given by Harvey $(1967 a, b)$ for the spectra of re-entry objects in ergs $/ \AA / \mathrm{sec}$ are not suitable, and integrated values crossing the whole 'profile' of the line are better. It is better to deal with the total brightness of the line using either a sufficiently wide densitometer slit (taking practically all the line image and the immediate vicinity), or quite simple visual estimates by comparison of suitable zero-order star images. If the zero-order star images are used, the selective transmission of the grating must be computed for different wavelengths of the stellar spectrum, and the values integrated. The spectral irradiance in ergs $/ \AA /$ sec is of course necessary to use for any absolute intensity data on the continuum or quasi-continuum (molecular) radiation.

It is a pity that the majority of meteor spectra photographed possess no additional geometrical and velocity data on the trajectory. It is very desirable to observe the meteor trajectory by standard double-station cameras (two fixed and one driven), or to use any other instrumental arrangement which enables one to obtain the complete data for the majority of the spectra photographed.

\section{Elements Observed}

The following elements are observed in spectra of the meteor head: neutral atoms: FeI, MgI, NaI, CaI, MnI, CrI, Ali, Niı, Hi, OI, Ni; ionized atoms: CaII, MgII, SiII, FeII, NiI, OII. There are some additional elements (CoI, SrI, SrII, BaI, BaII, SiI) which are sometimes identified but their presence is doubtful. New lines of CaII, FeI and FeII were added recently in the ultraviolet spectra photographed by Halliday (1967).

The meteor spectrum changes with the velocity and with the luminosity of the body. The CaII lines $\mathrm{H}$ and $\mathrm{K}$ are the main feature for fast meteors. Their relative 
intensity to lines of neutral elements (FeI) increases along the trajectory and is extremely high in the meteor flares. The same seems to hold for the MgII line 4481 with some limitation, given later. The $\mathrm{NaI}, \mathrm{MgI}_{\text {and }} \mathrm{Fe}$ l lines are dominant in spectra of slow meteors. This fact is due to the velocity effect and not to changes in the composition. Few meteors were observed with a spectrum consisting only of iron lines (Halliday, 1960b; Ceplecha, 1966b, 1967a). The dispersion of $58 \AA / \mathrm{mm}$ for such an iron meteor spectrum observed by Ceplecha (1966b) makes the identification of only iron lines quite sure. This is clearly caused by the composition of the body, which is probably quite analogous to the iron meteorites of small dimensions.

\section{Wake and Train Spectra}

There is no great difference between the meteor head and wake spectra, if considering element identifications only. As the wake spectra show substantially less excitation than the head spectra (close to $2000^{\circ} \mathrm{K}$ ), only the lines corresponding to the lowenergy states are recorded.

Diffusion effects were observed in the wake spectrum of a Geminid by Halliday (1963). A split of the wake into red and violet lines diverging backwards seems to be a rather exceptional type of wake. A diffusion coefficient 5 orders of magnitude higher than normal is against any explanation by diffusion. The instantaneous exposures of wakes in direct total light by Babadžanov and Kramer (1965) show just the opposite: the wake converges backwards. The 'diffusion' phenomenon observed by Halliday could in some way be connected with the pronounced flicker (50 to 300 cycles/sec) of the brightness of that meteor. The zigzag shape of meteor spectral lines, often observed, especially in the high-dispersion spectrograms, could also be connected with this split type of wake.

A quite exceptional position among the spectral features is shown by the forbidden oxygen line 5577 (Halliday, 1958, 1960a; Millman, 1960). It is present mainly in fast meteors and the radiation appears substantially higher $(10 \mathrm{~km})$ than the rest of the spectrum (Millman, 1963). Its identification is quite sure due to its duration of the order of $1 \mathrm{sec}$, quite different from other lines of meteor spectra. Russell (1963) found an inverse correlation between the intensity ratio of the $\mathrm{K}$ line of $\mathrm{Ca}$ II at 3830, and the $\mathrm{O}$ I line 5577 to the $\mathrm{D}$ lines of $\mathrm{Na}$ I. The forbidden oxygen line is probably caused by atmospheric oxygen, but there is no definitive decision of the mechanism of its origin. The most probable seems the dissociative recombination of $\mathrm{O}_{2}^{+}$by free electrons. The solar influence on the line production through corpuscular radiation could be present. There is a possibility that the radiation in the line 5577 is connected with the radar head echo (Millman, 1963), and with the typical visual meteor trains of short duration (Hughes, 1959). It is not excluded that the 'beginning height' groups, found by Ceplecha $(1966 a, 1967 b)$, are connected with the forbidden oxygen line appearance. 


\section{Quantitative Analysis of Meteor Spectra}

The first computation of excitation temperatures for meteor spectra was published by Millman $(1932,1935)$. The temperature range, found from $1680^{\circ}$ to $3220^{\circ} \mathrm{K}$, was revised by Cook (1955) but only small changes were necessary.

Cook and Millman (1955; Millman and Cook, 1959) made quantitative analyses of two low-dispersion grating spectra. They proceeded to the numbers of atoms in the upper state of several lines, assuming that the radiation was optically thin. The order of the upper state population was from $10^{15}$ to $10^{17}$.

Ceplecha $(1964,1965,1967 a)$ applied the method of the emission curve of growth for meteor spectra of high dispersion. Thus the complete quantitative spectral analysis for iron, and some simplified analysis for other elements, were made. Values found for each particular point of the meteor trajectory are: excitation temperature, total number of all atoms of the particular element inside the complete luminous volume, radiation area (surface of the luminous volume), damping constant. Dimensions of the luminous volume can be derived from the radiation area.

Two of the spectra studied are quite usual for slow meteors, and the computed abundances of the elements are approximately those of an average stony meteorite. The third spectrum is exceptional, and only lines of FeI and FeII (with traces of NiI and CoI lines) were found (Ceplecha, 1966b).

The resulting radiation area in all cases is greater than what corresponds to any spherically symmetrical model. Thus a cylindrical model was used and the radiation area then gives $R^{2}+R l$, where $R$ is the radius and $l$ the length of the luminous cylinder. Combination of this value with the dimensions of the image of optically thick lines in the focal plane yields the separation of $R$ from $l$. A typical ratio of $l / R$ was found between 10 and 100 for -5 to -10 meteor magnitudes. The ratio increases with the brightness. The excitation temperature determined from the FeI lines was found in the range from $2900^{\circ} \mathrm{K}$ to $4090^{\circ} \mathrm{K}$ and decreased with the increasing length of the luminous volume, being thus less in the brightest parts of the meteor trajectory. This effect is caused by the wake spectrum superposed on the head spectrum. An excitation temperature of the pure wake of $2400^{\circ} \mathrm{K}$ was found. The changes of excitation temperature behind the head were computed from $T=T(1) \times z^{q}$, where $q$ was found to be $-0 \cdot 12$ for a meteor velocity of about $30 \mathrm{~km} / \mathrm{sec}$. Lines of ionized iron were present at one point of the iron meteoroid trajectory ( $-10 \cdot 3$ magnitude). Using these and the FeI lines at the same point, the local thermodynamic-equilibrium conditions were checked. The FeI atoms were present in an idealized cylindrical volume of $12 \mathrm{~m}$ in diameter and $540 \mathrm{~m}$ in length, with an average concentration of $\log n(\mathrm{FeI})=15.54$ at an average temperature $3195^{\circ} \mathrm{K}$. Assuming the same diameter of the luminous cylinder, its length for the FeII atoms at the same time was only $7 \mathrm{~m}$, with an average concentration practically identical with the electron concentration $\log n(\mathrm{FeII})=\log$ $n(e)=15.21$ at an average temperature $5350^{\circ} \mathrm{K}$. Inside this smaller volume the con- 
centration of the FeI atoms was $\log n(\mathrm{FeI})=16.46$ on an average. The radiation damping was caused by the electron collisions with the iron atoms. The corresponding effective cross section was found, $\log \sigma=-11.9$. The exchange of energy by collisions of gaseous iron atoms with the air atoms cannot explain the observed length of the luminous volume. As the fragmentation of an iron meteoroid is improbable, the main ablation process was probably a spraying of the droplets from the molten surface of the body. It is significant that the wake phenomenon of this iron meteoroid did not differ from the wake phenomenon of other meteors.

The assumption of an average constant temperature for the computation of theoretical curves of growth (Ceplecha, 1964) is in contradiction to the results of the application of these theoretical curves to the meteor spectra, which clearly points to a change of temperature in the luminous volume, as given above. This stimulated Oleak (1966) to compute the theoretical curve of growth with a more complicated model, using temperature as one of independent variables. The practical application of this theory to meteor spectra is not easy, due to the enormous computer time necessary.

There are some major problems in the present analyses of meteor spectra by the emission curve of growth. The most striking is the great total mass resulting, which is one or two orders higher than the mass derived by the usual procedure from the light curve. I checked this disagreement, assuming optically thin radiation for the iron meteoroid, which ought to give the least mass from the curve of growth. But it is definitely not possible to construct the emission curve of growth (identical with a $45^{\circ}$ inclined straight line) for optically thin lines. I also tried different parts of the emission curve of growth as starting values, but a better solution than that given above was not found. A possible explanation of the mass discrepancy could be simply in the fact that each line has its own luminous volume, low-excitation lines a bigger one and high-excitation lines a smaller one. This corresponds to the observed luminous volumes of $\mathrm{FeI}$ and FeII of the iron meteoroid. Then the excitation temperature is given mainly by the bright low-excitation lines, and is lower than it ought to be for the faint high-excitation lines. These faint lines mainly determine the level of optically thin radiation, and thus the number of all atoms inside the luminous volume. This effect could explain even more than two orders of discrepancy in meteor masses. Assuming this effect to be working, the lines would show a tendency for an equalization of the intensity relative to the computed Planck continuum over a large interval of $\log g f \lambda-\Theta E_{1}$, which is actually observed. It is desirable to study this effect in more detail.

\section{The Luminous Equation}

It is obvious that to compute with the luminous equation $I=-\tau / 2 \times \mathrm{d} m / \mathrm{d} t v^{2}$, some additional relation, a dependence of $\tau$ on $v$, must be used. Integrating this equation, it has been assumed that representative meteor masses are obtained. There are some distinct indications that this equation is not valid. Kolomiec (1966) criticized an 
analogous equation used for the computation of electron density from radar echoes, and in conclusion he points out that the same holds for the luminous equation. The results from meteor spectra using the emission curve of growth show great variability in the luminous volume. The same was observed by Babadžanov and Kramer (1965) using very short exposures giving instantaneous images of the meteor.

The total energy of a meteor radiated inside a given spectral range, assuming constant excitation temperature, is proportional to the total amount of all the gaseous atoms inside the luminous volume. The time change of the number of these gas atoms is equal to the number of atoms added from the body to the luminous volume minus the number of atoms which leave the luminous volume. But the luminous equation includes only the gas atoms inside the luminous volume assumed to come from evaporation of the body. If an ablation process, the spraying of droplets or crumbling to fragments, is present, then the discrepancy is even greater. In this case the sources of the gas atoms are directly inside the luminous volume. Theoretically speaking, with the preceding considerations in mind, it is possible to proceed to well-known pulsations and flares observed in meteors, using only monotonic functions for the time change of the incoming and outgoing gas mass. It is highly desirable to study this problem in detail.

\section{Excitation and Ionization Changes}

The increase of excitation and ionization with velocity, and along the meteor trajectory, was explained by Cook (1955) as following the increase of the ratio of the characteristic dimensions of the body to the mean free path of an evaporating atom. Rajchl $(1963,1964)$ used the reciprocal modified Knudsen number $B$ for the study of excitation and ionization conditions. He found that the appearance of the ionized lines (CaII) is connected with the beginning of the slip flow conditions for the body and the appearance of the neutral lines with the beginning of the transition flow regime. On the other hand there are some definite indications that excitation decreases with increasing meteor brightness, especially in meteor flares (Millman, 1935; Cook, 1955; Russell, 1960b; Ceplecha, 1965, 1967a). This contradiction could be easily explained, if we assume that it is not possible to separate the wake radiation from that of the meteor head. Pure wake spectra are known between the breaks of a rotating shutter, and very low excitation was found for them. Thus meteors with strong wakes, especially in flares, could exhibit an apparent decrease of excitation due to the superposition of wake and head spectra. This idea was verified by Ceplecha for the iron meteoroid spectrum analysed by the method of the emission curve of growth. Each emission line can originate in a column of different length. The lines of low excitation potential are at any given instant of greater length and thus correspond to lower average-excitation temperatures (due to the decrease along the luminous column) than the lines of high excitation potential, which originate almost entirely inside the meteor head. 
Sometimes ionization changes are studied using the MgII line 4481. But this line is always blended with the FeI line 4482 of multiplet No. 2 (Moore, 1945). Thus the increase of brightness of this line in the flares is more likely associated with the superposed wake spectrum, which has a relatively bright Fe 4482 line due to the very low excitation potential. There is no identification of any MgII 4481 line without a blend, the presence of MgII being thus only probable. The intensity of the FeI 4482 line, if computed from the emission curve of growth for meteors with velocities of about $30 \mathrm{~km} / \mathrm{sec}$, is quite consistent with the observed intensity and the blend with MgII is not necessary. The wavelength measured absolutely and independently in the $3 \mathrm{rd}$, 4th and 5 th orders of the $11-38 \AA / \mathrm{mm}$ spectrum is $4481 \cdot 90 \pm 0 \cdot 18 \AA$, which clearly points to the absence of MgII in this spectrum.

\section{Spectra of Re-Entry Objects}

The comparison of re-entry object spectra with meteor spectra is of great importance in meteor spectroscopy and for the determination of meteor masses. The known initial mass of a re-entry object can be compared with the mass computed from the emission curve of growth, and some conclusions on the application of this method to meteor spectra can be drawn. Harvey $(1967 a, b)$ published spectra of three artificial meteoroids with a velocity of about $10 \mathrm{~km} / \mathrm{sec}$ and initial mass of about 1 gr. Only one case has sufficient dispersion for an analysis by the curve of growth, i.e. the Arcas-Margo iron dust ball meteor. There are some problems with the absolute intensity scale given by Harvey per $\AA$ of the profiles, which certainly are not real profiles of the lines. Ceplecha's analysis of this meteor gives the result that about $10 \%$ of the $1.5 \mathrm{gr}$ iron mass (density $0.05 \mathrm{gr} / \mathrm{cm}^{3}$ ) was converted into gas with an excitation temperature of $4300^{\circ} \mathrm{K}$ and damping constant $\log \gamma=9 \cdot 5$. This is different from that expected from meteor spectra at a velocity $10 \mathrm{~km} / \mathrm{sec}$. Thus, it can be concluded that practically all the evaporation and excitation observed in Harvey's spectrograms of the Arcas Margo meteor originates from the detonation of the shaped charge. It is desirable, in planning future experiments of this kind, to avoid all evaporation and excitation except what occurs in free flight.

During the last few years Millman (1967) has been engaged in a study of the general characteristics of meteor spectra, the spectra of re-entry objects, and spectra of other high-atmosphere phenomena.

\section{Molecular Spectra}

The 1st, and sometimes the 2 nd positive systems of the $\mathrm{N}_{2}$ molecule are normally identified in meteor spectra (Cook and Millman, 1955, 1959; Russell, 1960). I can present here some preliminary results of meteor spectrum S 1132 (meteor No. 38421), which exhibits strong molecular bands due to a strong wake. This grating spectrum 
has a dispersion of about $57 \AA / \mathrm{mm}$ and belongs to a meteor with $a=5 \cdot 1, e=0 \cdot 82$, $i=49^{\circ}, v_{\propto}=32.5 \mathrm{~km} / \mathrm{sec}$, beginning height $97 \mathrm{~km}$, and with a very bright terminal flare at a height of $76 \mathrm{~km}$. The spectrum of the flare is a superposition of the meteor spectrum on a wake spectrum of approximately the same total brightness. This fact is evident from one additional prism spectrum of the same meteor. Fortunately, the prism spectrum is cut at the flare by the rotating shutter, showing thus the wake spectrum at low dispersion. The grating spectrum is cut by the rotating shutter outside the terminal flare, being thus a mixture of both the wake and flare spectrum. There were found about 430 different atomic emission lines, together with a strong 'continuum' emission from $5500 \AA$ to $6600 \AA$. The atomic spectrum is quite usual with Feı, Mgı, NaI, CaII, CaI, SiII, Alı, Hı, MnI, Cri, Ni and CoI. The lines were measured in a measuring engine and a densitometer tracing was also made. If the atomic emissions with good identifications are subtracted from the tracing, more than 100 band heads are observed in the continuum emission. This makes the identification of molecular bands more certain. About 20 lines which were measured with the measuring engine, and for which an atomic identification failed or was very improbable (as for example AlIII), were definitely classified as band heads. Thus the following molecules have been provisionally identified, using as the main criterion the presence of a majority of the band heads to be expected for a particular molecule, some of them being present without blends. The total number of observed band heads, and the wavelengths (Pearse and Gaydon, 1950) of those heads without blends, are given below:

$\mathrm{N}_{2} 16$ heads $(5804,5755,5632+5633) ; \mathrm{FeO} 23$ heads $(6597,6524,6296,6180,6110$, $5975,5819,5790,5543)$; $\mathrm{MgO} 4$ heads $(6581,6312)$; $\mathrm{CaO} 17$ heads $(6362,6041), \mathrm{CN}$ 38 heads $(6632,6479,6456,6294,6272,6214,6206,6153,6147,5993,5849,5749$, $5722,5607,5591) ; C_{2} 9$ heads $(6599,6534,6480,5923,5636)$; CO 24 heads $(6620$, $6513,6383,6037,5837,5812,5779,5670) ; \mathrm{CO}^{+} 5$ heads $(6239,5970,5764,5694)$. This seems rather surprising, hence I shall try to publish the complete material, including a reproduction of the tracing, in the near future.

As all the molecular emissions were observed at the red end of the spectrum, there is a possibility that the same molecules could be responsible for the overwhelming red radiation of fainter meteors found by Ceplecha (1959), and verified by Davis (1963).

\section{Recommendation for Future Work}

(a) It is highly desirable to publish all the data of meteors with good dispersion spectra, i.e. height and velocity data, wavelengths, and relative or absolute intensities of lines, at one or more points of the trajectory.

(b) Good persistent train spectra by transmission grating are needed.

(c) Grating spectrographs with a rotating shutter for instantaneous exposures ought to be used. 
(d) A slit spectrum with a dispersion of the order of $1 \AA / \mathrm{mm}$ would be a big help for quantitative spectral studies.

(e) The forbidden oxygen line $5577 \AA$ ought to be studied by the technique developed by Halliday and Griffin (1963).

(f) Further quantitative analyses of meteor spectra are needed.

(g) The revision of the luminous equation, using meteor spectra, is highly desirable.

(h) The zigzag shape of spectral lines in meteor spectra calls for some explanation.

(i) Publication of data on the spectra of re-entry objects is highly desirable.

\section{References}

Babadžanov, P. B., Kramer, E. N. (1965) Astr. Zu., 42, 660.

Ceplecha, Z. (1959) Bull. astr. Inst. Csl., 10, 39.

Ceplecha, Z. (1961) Bull. astr. Inst. Csl., 12, 246.

Ceplecha, Z. (1963) Bull. astr. Inst. Csl., 14, 11.

Ceplecha, Z. (1964) Bull. astr. Inst. Csl., 15, 102.

Ceplecha, Z. (1965) Bull. astr. Inst. Csl., 16, 88.

Ceplecha, Z. (1966a) Bull. astr. Inst. Csl., 17, 96.

Ceplecha, Z. (1966b) Bull. astr. Inst. Csl., 17, 195.

Ceplecha, Z. (1967a) Bull. astr. Inst. Csl., 18, 303.

Ceplecha, Z. (1967b) Smithson. Contr. Astrophys. 11, 35.

Ceplecha, Z., Rajchl, J. (1963) Bull. astr. Inst. Csl., 14, 29.

Cook, A.F. (1955) J. atmos. terr. Phys., Spec. Suppl., $2,8$.

Cook, A. F., Millman, P. M. (1955) Astrophys. J., 121, 250.

Davis, J. (1963) Smithson. Contr. Astrophys., 7, 233.

Halliday, I. (1958) Astrophys. J., 128, 441.

Halliday, I. (1960a) Astrophys. J., 131, 25.

Halliday, I. (1960b) Astrophys. J., 132, 482.

Halliday, I. (1961) Publ. Dom. Obs., Ottawa, 25, 1.

Halliday, I. (1963) Smithson. Contr. Astrophys., 7, 161.

Halliday, I. (1967) private communication.

Halliday, I., Griffin, A.A. (1963) J. scient. Instrum., 40, 187.

Harvey, G.A. (1967a) NASA, Tech. Note D-3930.

Harvey, G.A. (1967b) NASA, Tech. Note D-3765.

Hirose, H. (1967) private communication.

Hughes, R.F. (1959) Smithson. Contr. Astrophys., 3, 79.

Kolomiec, A. R. (1966) Geomagn. Aeronom., 6, 943.

Millman, P. M. (1932) Ann. Harv. Coll. Obs., 82, 113.

Millman, P. M. (1935) Ann. Harv. Coll. Obs., 82, 149.

Millman, P. M. (1960) J. R. astr. Soc. Can., 54, 189.

Millman, P. M. (1963) J. R. astr. Soc. Can., 57, 89.

Millman, P.M. (1967) private communication.

Millman, P. M., Cook, A.F. (1959) Astrophys. J., 130, 648.

Millman, P.M., Halliday, I. (1961) Planet. Space. Sci., 5, 137.

Moore, C.E. (1945) Contr. Princeton Obs., 20.

Nasyrova, L.I., Nasyrov, G.A. (1966) Astr. Circ. Izdav. Bjuro astr. Soob̌̌č., 370.

Oleak, H. (1966) Astr. Nachr., 289, 157.

Pearse, R.W.P., Gaydon, A.G. (1950) The Identification of Molecular Spectra, Chapman and Hall, London.

Rajchl, J. (1963) Smithson. Contr. Astrophys., 7, 155.

Rajchl, J. (1964) Bull. astr. Inst. Csl., 15, 138. 
Russell, J.A. (1960a) Publ. astr. Soc. Pacific, 72, 291.

Russell, J.A. (1960b) Astrophys. J., 131, 34.

Russell, J.A. (1963) Smithson. Contr. Astrophys., 7, 171.

Russell, J.A. (1964) Meteoritics, 2, 117.

\section{DISCUSSION}

Halliday: In the case of the Geminid meteor with the split lines in the wake, my use of the word 'diffusion' was to indicate that meteoric atoms were rapidly distributed in the atmosphere. As mentioned later in my paper the phenomenon was probably more like an explosion than a normal diffusion process. Concerning $\lambda 4481$ of $\mathrm{Mg}$ II we have a high-dispersion spectrum of a Lyrid meteor where the shift between $\lambda 4482$ of FeI in the wake spectrum and $\lambda 4481$ of MgII can be seen, so there is no doubt that $\lambda 4481$ is a strong line in the head spectrum.

Ceplecha: I spoke about this 'split wake' only to point out that it is a rather exceptional case.

Millman: Could you tell me if there was any evidence of asymmetry in the tracings of the molecular band heads you have identified?

Ceplecha: Yes, there was. I will publish the complete tracing data for the red end of this spectrum as soon as possible.

Cook: Have you checked your assumption of local thermodynamic equilibrium by estimating excitation frequencies by collisions and comparing these with de-excitation frequencies by radiation?

Ceplecha: No. I proceeded in a different way, as described briefly in my survey paper, and in detail in my second paper on the iron meteoroid (1967a).

Whipple: I wish to congratulate Dr. Ceplecha on this remarkable spectrum. The presence of cometary bands is very significant both in suggesting a cometary origin for the meteoroid, and in showing that the molecules are carried in the solid meteoritic structure.

Bronsten: In your opinion, has the Planck continuous spectrum of the head shock wave of the meteoroid ever been observed?

Ceplecha: I know you wish to have some observational evidence of excitation temperatures and continuum radiation of the order of many ten thousands of degrees. But experience with the meteor spectra I have does not show any evidence of such shock-wave continuum radiation, probably due to the absorption of the air. 